\title{
User Association and Power Allocation for Multi-cell Non- Orthogonal Multiple Access Networks
}

DOI:

10.1109/TWC.2019.2935433

\section{Document Version}

Accepted author manuscript

Link to publication record in Manchester Research Explorer

\section{Citation for published version (APA):}

Wang, K., Liu, Y., Ding, Z., Nallanathan, A., \& Peng, M. (2019). User Association and Power Allocation for Multicell Non-Orthogonal Multiple Access Networks. IEEE Transactions on Wireless Communications, 1-1.

https://doi.org/10.1109/TWC.2019.2935433

\section{Published in:}

IEEE Transactions on Wireless Communications

\section{Citing this paper}

Please note that where the full-text provided on Manchester Research Explorer is the Author Accepted Manuscript or Proof version this may differ from the final Published version. If citing, it is advised that you check and use the publisher's definitive version.

\section{General rights}

Copyright and moral rights for the publications made accessible in the Research Explorer are retained by the authors and/or other copyright owners and it is a condition of accessing publications that users recognise and abide by the legal requirements associated with these rights.

\section{Takedown policy}

If you believe that this document breaches copyright please refer to the University of Manchester's Takedown Procedures [http://man.ac.uk/04Y6Bo] or contact uml.scholarlycommunications@manchester.ac.uk providing relevant details, so we can investigate your claim.

\section{OPEN ACCESS}




\title{
User Association and Power Allocation for Multi-cell Non-Orthogonal Multiple Access Networks
}

\author{
Kaidi Wang, Student Member, IEEE, Yuanwei Liu, Senior Member, IEEE, Zhiguo Ding, Senior Member, IEEE, \\ Arumugam Nallanathan, Fellow, IEEE, and Mugen Peng, Senior Member, IEEE
}

\begin{abstract}
In this paper, user association and power allocation are investigated in a non-orthogonal multiple access (NOMA)based multi-cell network. In order to perform successive interference cancellation (SIC) techniques for removing the intra-base station (BS) interference, the optimal decoding order is derived for all users associated with the same BS. In an effort to improve the system, a sum rate maximization problem is formulated by jointly designing user association and power allocation. Two game theory based algorithms are proposed to obtain the stable user structure by dividing users into different BSs' clusters, where the sub-optimal and global optimal solutions can be achieved. The properties of the proposed algorithms, including complexity, convergence, stability and optimality, are analyzed. Based on the quality-of-service (QoS) constraint, the closed-from solutions for power allocation are derived, and thus the expressions for the sum rate of all users in each cluster is obtained. Moreover, the case that the QoS threshold cannot be achieved by all users in each cluster is considered. Simulation results demonstrate that: i) the proposed user association algorithms and the closedform solutions for power allocation can significantly enhance the sum rate and outage probability; and ii) the proposed NOMA-based system is capable of achieving promising gains over the conventional orthogonal multiple access (OMA)-based framework in the multi-cell scenario.
\end{abstract}

Index Terms-Game theory, non-orthogonal multiple access (NOMA), power allocation, user association

\section{INTRODUCTION}

Multiple access (MA) techniques, as one of the key enabling techniques in the fifth generation (5G) wireless network, have received considerable attentions and extensive research interest. The existing MA technologies can be classified into orthogonal multiple access (OMA) and non-orthogonal multiple access (NOMA) approaches based on whether the users are served in orthogonal resource blocks [2]. NOMA as a scheme which allows non-zero cross correlation signals, can

The work of K. Wang and Z. Ding was supported by the UK Engineering and Physical Sciences Research Council (EPSRC) under grant number EP/P009719/2 and by H2020-MSCA-RISE-2015 under grant number 690750. The work of M. Peng was supported by the National Natural Science Foundation of China under grant number 61831002.

K. Wang and Z. Ding are with School of Electrical and Electronic Engineering, The University of Manchester, Manchester, M13 9PL, U.K. (email: kaidi.wang@postgrad.manchester.ac.uk; zhiguo.ding@manchester.ac.uk.

Y. Liu and A. Nallanathan are with Queen Mary University of London, London, E1 4NS, U.K. (email:\{yuanwei.liu, a.nallanathan\}@qmul.ac.uk).

M. Peng is with the Institute of Telecommunications, Beijing University of Posts and Telecommunications, Beijing, 100876, China. (email:pmg@bupt.edu.cn).

Part of this work has been published in IEEE International Conference on Communications (ICC), May, Kansas City, 2018 [1]. improve spectrum efficiency and provide massive connectivity [3]. In conventional OMA schemes, such as frequency division multiple access (FDMA) and time division multiple access (TDMA), the spectrum efficiency is low when the bandwidth resources are occupied by the users having poor channel conditions [4]. However, the users in NOMA systems are communicating in the same frequency, time and code with different power levels [5]. By carefully selecting the power allocation coefficients, the system throughput and the user fairness in NOMA systems can be guaranteed [6]. More particularly, the users with strong channel conditions are capable of removing the interference of weak users with the aid of successive interference cancellation (SIC) techniques [7]. The random allocated users in a cellular downlink NOMA system was studied in [8], which shows that NOMA can outperform traditional OMA in terms of the ergodic sum rate and outage probability. By investigating user paring in multiple scenarios, the authors in [9] have pointed out that both sum rate and individual rates in NOMA systems were greater than OMA. As a development, user pairing was also investigated in [10]. By comparing the different schemes of user pairing, it was indicated that the NOMA systems can consequently outperform OMA in spectral efficiency and system throughput. In [11], the user fairness was considered as the standpoint. The simulation results demonstrated that NOMA schemes can achieve the better fairness and efficiency than OMA.

\section{A. Related Works}

In existing research contributions, most of the papers have explored the potential of NOMA systems by considering user pairing/clustering and power allocation [12]-[17]. More particularly, user pairing was studied in [12], where the user with the weak channel condition was paired with the user with the strong channel condition when the minimum data rate was achieved. As the extension of user pairing, user clustering and power allocation were studied in [13] for improving the fairness in a downlink multi-input multi-output (MIMO)NOMA system. The impact of sub-carrier assignment and power allocation were studied in NOMA systems [14], where the formulated problems were jointly optimized with weight factors. Furthermore, the jointly designed user clustering and power allocation were investigated in both uplink and downlink NOMA systems [15], in which the formulated problems were respectively solved by a low-complexity algorithm and 
closed-form solutions. The downlink and uplink NOMA systems were also considered in [16], [17], where the presented joint cell association and power control problem was solved by proposed algorithms.

However, user pairing/clustering and power allocation in multi-cell NOMA systems are more challenging than that in single-cell NOMA systems. Specifically, in addition to the intra-BS interference caused by the co-channel interference of NOMA systems, the inter-BS interference caused by the un-associated BSs also needs to be take into consideration [18], [19]. In order to mitigate inter-BS interference, different technologies have been proposed to multi-cell NOMA systems [20]-[28]. Specifically, in [20], heterogeneous networks (HetNets) were adopted in multi-cell NOMA systems, where massive MIMO and NOMA were considered in macro and pico BSs, respectively. Hybrid HetNets were also applied in [21], [22], in which OMA and NOMA were exploited in different tiers. Coordinated multipoint (CoMP) transmission was discussed in [23], where the user located in the cell-edge area can receive signals from multiple BSs. As an extension of [23], power allocation was optimized at each coordinating BS individually to reduce the huge computational complexity in [24]. By adopting beamforming techniques, inter-BS interference was partly removed in the multi-cell mmWave-NOMA network [25]. Furthermore, the negative impact of inter-BS interference can also be moderated by utilizing resource blocks to serve the users at different time or frequency domains [26][28].

\section{B. Motivation and Contributions}

As mentioned above, the multi-cell NOMA system has been extensively explored with various technologies, but, to be best of our knowledge, the NOMA-based homogeneous multi-cell networks have not been researched. More specifically, the implementation of NOMA brings more sophisticated co-channel interference to the existing networks, and such distinct characteristics necessitate the redesign of user association. To further enhance the performance of NOMA networks, the optimization of power allocation coefficients should be considered. It is a meaningful and challenging problem for simultaneously partitioning users into different clusters and maximizing the sum rate with different power levels. However, the complete systematic performance of multi-cell NOMA systems by jointly designing user clustering and power allocation is still not well studied due to the high computational complexity, which motivates this work. In the previous works, the user clustering problem was only considered in the two-user case [21], [23], [27], and the power allocation minimization problem was solved in a random user structure [22], [26], where these obtained solutions result in the significant computational complexity [28]. In this paper, the users connected to the same BS are treated as a cluster and receive signals with NOMA principles. The power allocation coefficients in NOMA schemes are iteratively optimized. The main contributions of this paper can be cited as follows:

- A downlink multi-cell NOMA network is investigated, where users are allocated to multiple clusters controlled by different BSs. In particular, in each cluster, the optimal decoding order for SIC techniques is first derived. Then, based on the optimal decoding order, the user association and power allocation problems are jointly designed and iteratively solved.

- With the aid of game theory, two user association algorithms, namely, preference relation based algorithm (PRA) and simulated annealing based algorithm (SAA), are proposed. More particularly, PRA provides a low complexity approach for obtaining the suboptimal solution, while SAA can yield the global optimal solution with a large number of iterations. The properties of both algorithms, including complexity, convergence, stability, and optimality, are analyzed.

- For the power allocation problem, the closed-form expressions for the sum rate are derived and employed at each iteration of the proposed user association algorithms. In particular, two types of quality-of-service (QoS) constraints are considered while developing the solutions: in the first case, all users' rate requirements need to be satisfied; in the second case, only part of users' minimum rates need to be realized.

- Simulation results are presented to illustrate that PRA with the fixed power allocation can achieve the nearoptimal solutions with much lower complexity compared with the proposed global optimal algorithm. It is also demonstrated that user association and power allocation in the NOMA-based framework can achieve better performance in the sum rate and outage probability than the OMA-based framework.

\section{Organization}

The rest of the paper is organized as follows. In Section II, a NOMA-based downlink system with multiple BSs and users is demonstrated. In Section III, an optimization problem is formulated for maximizing the sum rate of the multicell NOMA system, and divided into user association and power allocation problems. In Section IV, two coalitional game based algorithms are proposed for solving the formulated user association problem. In Section V, the closed-form expressions for power allocation coefficients are derived. In Section VI, simulation results are presented with the analysis. Finally, a conclusion is summarized in Section VII.

\section{NETWORK MODEL}

In this section, a NOMA-based multi-cell framework is presented, in which the user association scenario is investigated and the optimal decoding order for SIC techniques is analyzed.

\section{A. System Description}

Consider a downlink multi-cell NOMA system with $K$ BSs and $N$ users, where $N>K$. The BSs are located at the fixed points of a disc $D$ with radius $R_{D}$, and users are uniformly distributed within the disc. The collections of all BSs and users are denoted by $\mathcal{K}=\{1,2, \cdots, K\}$ and $\mathcal{N}=\{1,2, \cdots, N\}$, respectively. It is assumed that the individual users can be 


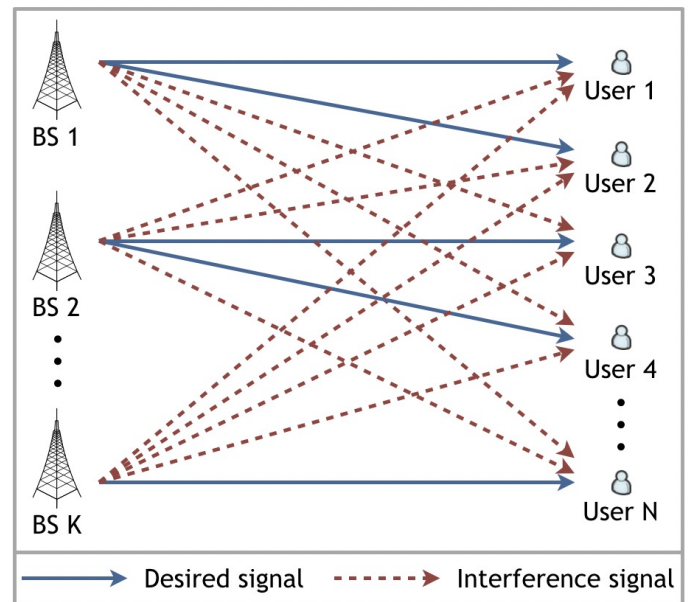

Fig. 1: An illustration of the proposed downlink multi-cell NOMA network.

associated with one BS at the same time. The data of users is shared among multiple BSs, but each BS only transmits the superposition signal of the connected users. It is also assumed that the total transmit power of each BS is equal ${ }^{1}$. The users associated with the same BS are considered as a cluster, hence, there are $K$ clusters formed in this paper. The user structure $\mathcal{S}$ is the collection of all clusters, i.e., $\mathcal{S}=\left\{S_{1}, S_{2}, \cdots, S_{K}\right\}$, where any cluster $S_{k}$ is formed by BS $k$. The NOMA scheme is employed in the proposed system, which implies that all BSs transmit signals in the same frequency and time slot. Or in other words, for any user, both desired and interference signals are received, as shown in Fig. 1.

\section{B. Signal Model}

In the proposed multi-cell NOMA system, two types of interferences should be considered. Firstly, the inter-BS interference is generated by the signals transmitted from the un-associated BSs. Secondly, the intra-BS interference is produced by the co-channel interference of NOMA schemes. Therefore, for any cluster $S_{k}$, the received signal at the $n$ th user associated with BS $k$ can be expressed as follows:

$$
\begin{aligned}
y_{k, n}= & \sum_{i=1}^{K} \sum_{j=1}^{\left|S_{i}\right|} h_{i, n} \sqrt{p_{i, j}} s_{i, j}+n_{k, n} \\
= & \underbrace{\sum_{i=1, i \neq k}^{K} \sum_{j=1}^{\left|S_{i}\right|} h_{i, n} \sqrt{p_{i, j}} s_{i, j}}_{\text {inter-BS interference }}+\underbrace{h_{k, n} \sum_{j=1, j \neq n}^{\left|S_{k}\right|} \sqrt{p_{k, j}} s_{k, j}}_{\text {intra-BS interference }} \\
& +h_{k, n} \sqrt{p_{k, n}} s_{k, n}+n_{k, n},
\end{aligned}
$$

where $h_{k, n}$ is the channel between BS $k$ and the $n$-th user in cluster $S_{k}, p_{k, n}$ is the power allocation coefficient of the $n$-th user, $s_{k, n}$ is the signal transmitted from BS $k$ to the $n$-th user, and $n_{k, n}$ is the additive noise. Additionally, the channel coefficient is defined by $\left|h_{k, n}\right|^{2}=\left|\hat{h}_{k, n}\right|^{2} L\left(d_{k, n}\right)$,

\footnotetext{
${ }^{1}$ Note that it is possible to use different transmit powers at different BSs. Resource allocation with such a different consideration is an important direction for future research, but it is out of the scope of this paper.
}

where $\hat{h}_{k, n} \sim \mathcal{C N}(0,1)$ is the small-scale fading coefficient from BS $k$ to the $n$-th user in cluster $S_{k}, L\left(d_{k, n}\right)=\eta d_{k, n}^{-\alpha}$ is the large-scale path loss, $d_{k, n}$ is the distance between BS $k$ and the $n$-th user, $\eta$ is the frequency dependent factor, and $\alpha$ is the path loss exponent. In NOMA schemes, the users associated with the same BS are served at different power levels. In any cluster $S_{k}$, the power allocation coefficients of all users associated with BS $k$ satisfy $\sum_{i=1}^{\left|S_{k}\right|} p_{k, i}=1$.

Due to the high density of BSs and users, the interference in the proposed system, especially the inter-BS interference, is very severe. The commonly used method for avoiding the inter-BS interference, such as fractional frequency reuse (FFR), is conflicting with NOMA schemes [18]. Therefore, in this paper, the inter-BS interference is treated as noise and the SIC technique is utilized to reduce the intra-BS interference. By employing SIC techniques, users with strong channel conditions need to detect and remove the signals of the users with weak channel conditions, and the weak users treat the signals of strong users as noise [29]. Without loss of generality, the users in each cluster are ordered based on their channel conditions, where the user with the best channel quality is set as the last user in the cluster. For the $n$-th user in any cluster $S_{k}$, where $i<n<j$, it can decode the signal of the $i$-th user, and treat the signal of the $j$-th user as noise. The inter-BS interference of the $n$-th user in cluster $S_{k}$ can be expressed as follows:

$$
I_{k, n}=\sum_{i=1, i \neq k}^{K} \sum_{j=1}^{\left|S_{i}\right|}\left|h_{i, n}\right|^{2} p_{i, j}=\sum_{i=1, i \neq k}^{K}\left|h_{i, n}\right|^{2} .
$$

It can be found that the inter-BS interference is fixed for the given user when the transmit power is equal for all BSs, and hence, the complexity for performing SIC in the proposed multi-cell NOMA system is similar as it in the conventional single-cell NOMA network. By allocating users into different clusters, the complexity for performing SIC techniques in each cluster is significantly reduced. By utilizing SIC techniques, the $n$-th user can receive the signal with the following signalto-interference-plus-noise ratio (SINR):

$$
S I N R_{k, n}=\frac{\left|h_{k, n}\right|^{2} p_{k, n}}{\left|h_{k, n}\right|^{2} \sum_{i=n+1}^{\left|S_{k}\right|} p_{k, i}+I_{k, n}+\frac{1}{\rho}},
$$

where $\rho=P_{t} / \sigma^{2}$ is the transmit signal-to-noise ratio (SNR), $P_{t}$ is the transmit power, and $\sigma^{2}$ is the variance of the additive white Gaussian noise (AWGN). For the last user in cluster $S_{k}$, it can remove all intra-BS interference by applying SIC techniques. Assume that there are $t$ users in cluster $S_{k}$, i.e., $\left|S_{k}\right|=t$, the $t$-th user can decode its own signal with the following SINR:

$$
S I N R_{k, t}=\frac{\left|h_{k, t}\right|^{2} p_{k, t}}{I_{k, t}+\frac{1}{\rho}},
$$

In this paper, it is assumed that the application of SIC is carried out at each user, which indicates that one user can always decode the signals of the users before it in the sequence. Assume that $m$ and $n$ are two users in cluster $S_{k}$, where the $n$-th user's channel condition is better than the $m$ th user, i.e., $m<n$. Based on [8] and [29], the condition of 
SIC is that the rate of the $n$-th user to decode the $m$-th user's signal is no less than the rate of the $m$-th user for decoding its own signal, i.e.,

$$
R_{k, n \rightarrow m} \geq R_{k, m \rightarrow m} .
$$

If above inequality is not satisfied, the SIC technique cannot be perfectly performed at the $m$-th user, and hence, the remaining intra-BS interference should be included. In this case, the achievable data rate of the $m$-th user is reduced. According to [8], the data rate of the $m$-th user should be constrained by the rate of the $n$-th user to decode the $m$-th user's signal, i.e., $R_{k, m \rightarrow m}=R_{k, n \rightarrow m}$. By considering all users after the $m$-th user, the data rate of the $m$-th user in cluster $S_{k}$ is limited by the subsequent users. The individual rate of the $m$-th user can be expressed as follows:

$$
R_{k, m}=\min \left\{R_{k, j \rightarrow m} \mid \forall j \geq m\right\},
$$

where the rate of the $j$-th user to decode the $m$-th user's signal is given by

$$
R_{k, j \rightarrow m}=\log _{2}\left(1+\frac{\left|h_{k, j}\right|^{2} p_{k, m}}{\left|h_{k, j}\right|^{2} \sum_{i=m+1}^{t} p_{k, i}+I_{k, j}+\frac{1}{\rho}}\right) .
$$

Hence, the achievable data rate of the $m$-th user in cluster $S_{k}$ can be expressed as follows:

$$
R_{k, m}=\min \left\{\log _{2}\left(1+\frac{\left|h_{k, j}\right|^{2} p_{k, m}}{\left|h_{k, j}\right|^{2} \sum_{i=m+1}^{t} p_{k, i}+I_{k, j}+\frac{1}{\rho}}\right) \mid \forall j \geq m\right\} .
$$

In other words, the data rate of the $m$-th user is equal to the minimum rate of the users after the $m$-th user to decode the $m$-th user's signal.

\section{Optimal Decoding Order}

In order to avoid the decrease of the achievable data rate, the decoding order of all users in each cluster should be determined. The conventional decoding order in single-cell NOMA systems, i.e., the order of channel gains, cannot be employed in the proposed system, since the inter-BS interference should be considered as part of channel conditions. In [28], user scheduling was jointly designed and iteratively executed in the user association algorithm. As a result, the computational complexity of user association is significantly increased. In this subsection, an optimal decoding order is derived, which can efficiently increase the individual rate of each user.

It can be found from Eq. (8) that the $m$-th user's data rate is partially decided by the channel conditions and interference of the users after it. By equivalent transformation, the following equation can be obtained:

$$
R_{k, m}=\log _{2}\left(1+\frac{p_{k, m}}{\sum_{i=m+1}^{t} p_{k, i}+\max \left\{\frac{\rho I_{k, j}+1}{\rho\left|h_{k, j}\right|^{2}} \mid \forall j \geq m\right\}}\right) .
$$

For maximizing the individual rate of each user in cluster $S_{k}$, the users should be ordered based on their channel conditions and inter-BS interference. The optimal decoding order of all users in cluster $S_{k}$ is a sequence as follows:

$$
\max \left\{\frac{\rho I_{k, j}+1}{\rho\left|h_{k, j}\right|^{2}} \mid \forall j \geq 1\right\} \geq \cdots \geq \max \left\{\frac{\rho I_{k, j}+1}{\rho\left|h_{k, j}\right|^{2}} \mid \forall j \geq t\right\} .
$$

The sequence in (10) is always satisfied if the following decoding order holds:

$$
\frac{\rho I_{k, 1}+1}{\rho\left|h_{k, 1}\right|^{2}} \geq \cdots \geq \frac{\rho I_{k, t}+1}{\rho\left|h_{k, t}\right|^{2}} .
$$

Based on the optimal decoding order, the condition of inequality (5) is always satisfied. The achievable data rate of the $m$-th user in cluster $S_{k}$ can be presented as follows:

$$
R_{k, m}=\log _{2}\left(1+\frac{\left|h_{k, m}\right|^{2} p_{k, m}}{\left|h_{k, m}\right|^{2} \sum_{i=m+1}^{t} p_{k, i}+I_{k, m}+\frac{1}{\rho}}\right) .
$$

Therefore, for raising the achievable data rates, the optimal decoding order in the proposed multi-cell NOMA system can be presented as follows:

Remark 1. In any cluster $S_{k}$ with $t$ users, the data rate of the $n$-th user in the multi-cell NOMA system is given by

$$
R_{k, n}=\log _{2}\left(1+\frac{\left|h_{k, n}\right|^{2} p_{k, n}}{\left|h_{k, n}\right|^{2} \sum_{i=n+1}^{t} p_{k, i}+I_{k, n}+\frac{1}{\rho}}\right),
$$

if all users in cluster $S_{k}$ are ordered based on the following sequence:

$$
\mathcal{Q}\left(S_{k}\right) \triangleq \frac{\rho I_{k, 1}+1}{\rho\left|h_{k, 1}\right|^{2}} \geq \frac{\rho I_{k, 2}+1}{\rho\left|h_{k, 2}\right|^{2}} \geq \cdots \geq \frac{\rho I_{k, t}+1}{\rho\left|h_{k, t}\right|^{2}} .
$$

\section{PRoblem Formulation}

For the sake of improving the performance of the proposed multi-cell NOMA system, an optimization problem is formulated for maximizing the sum rate. User clustering and power allocation are considered as efficient methods to enhance the performance of NOMA systems. That is, the users are associated with BSs by allocating them to different clusters, and the BSs transmit the signals to the associated users at different power levels. By optimizing the user structure and power allocation coefficients, the sum rate maximization problem can be formulated as follows:

$$
\begin{aligned}
\max _{\{\mathbf{x}, \mathbf{p}\}} & \sum_{i=1}^{K} \sum_{j=1}^{N} x_{i, j} R_{i, j} \\
\text { s.t. } & \mathcal{Q}\left(S_{i}\right), \forall i \in \mathcal{K}, \\
& R_{i, j} \geq \gamma, \forall i \in \mathcal{K}, \forall j \in S_{i}, \\
& x_{i, j} \in\{0,1\}, \forall i \in \mathcal{K}, \forall j \in \mathcal{N}, \\
& \sum_{i=1}^{K} x_{i, j}=1, \forall j \in \mathcal{N}, \\
& 0 \leq p_{i, j} \leq 1, \forall i \in \mathcal{K}, \forall j \in \mathcal{N}, \\
& \sum_{j=1}^{\left|S_{i}\right|} p_{i, j}=1, \forall i \in \mathcal{K},
\end{aligned}
$$

where $\mathbf{x}$ is the collection of all user association indicators, and $\mathbf{p}$ is the collection of all power allocation coefficients. More particularly, constraint (15a) is the optimal decoding 
order of the users in each cluster, which ensures that any user can successfully decode the signals of the users with weaker channel conditions. Constraint (15b) is the QoS threshold, where $\gamma$ is the minimum data rate of the individual users. Constraint (15c) is the value of the user association indicators, $x_{k, i}=1$ indicates that user $i$ is associated with BS $k, x_{k, i}=0$ otherwise. Constraints (15d) indicates that any user can only connect to one BS at the same time. The conditions of power allocation coefficients are presented in constraints (15e) and (15f).

It can be found that the formulated problem is a mixed integer linear programming (MILP) problem. More particularly, even if the power allocation coefficients are fixed, the user association related problem is still a non-deterministic polynomial-time (NP)-complete, since it subsumes a 0-1 linear program [30], [31]. Additionally, the non-convex problem is difficult to transform to a convex problem, since the channel conditions of users are varied by associating with different BSs. Therefore, the formulated optimization problem (15) is decoupled into a user association problem and a power allocation problem.

\section{A. User Association Problem}

The user association problem is formulated to obtain the stable user structure which can improve the performance of the proposed multi-cell NOMA system. By optimizing the indicators $\mathbf{x}$, the user association problem is formulated as follows:

$$
\begin{aligned}
\max _{\{\mathbf{x}\}} & \sum_{i=1}^{K} \sum_{j=1}^{N} x_{i, j} R_{i, j} \\
\text { s.t. } & \mathcal{Q}\left(S_{i}\right), \forall i \in \mathcal{K}, \\
& x_{i, j} \in\{0,1\}, \forall i \in \mathcal{K}, \forall j \in \mathcal{N}, \\
& \sum_{i=1}^{K} x_{i, j}=1, \forall j \in \mathcal{N} .
\end{aligned}
$$

In the user association problem, users connect to BSs for increasing the sum rate. In constraint (16a), the optimal decoding order is adopted in the user association problem, which indicates that the users are ordered in each given cluster. The QoS constraint is not included in the user association problem, since the existing of the target rate will severely restrict the swaps of users and affect the obtaining of the global optimal solutions. Consider a situation with extremely large number of users, not all users' QoS thresholds can be achieved. By considering the QoS constraint in this situation, some users cannot join in any cluster because their target rates are not achieved in these clusters, and the user structure cannot be changed in this situation. Without the QoS constraint, more users can swap between different clusters in the user association problem, and hence, the user structure can converge to a better result. Therefore, the QoS constraint is only considered in the power allocation problem in this paper, where the users' QoS thresholds can be achieved by adjusting power allocation coefficients.

\section{B. Power Allocation Optimization Problem}

In this subsection, the power allocation coefficients are optimized for enhancing the sum rate. The power allocation problem is formulated as follows:

$$
\begin{array}{cl}
\max _{\{\mathbf{p}\}} & \sum_{i=1}^{K} \sum_{j=1}^{\left|S_{i}\right|} R_{i, j} \\
\text { s.t. } & R_{i, j} \geq \gamma, \forall i \in \mathcal{K}, \forall j \in S_{i}, \\
& 0 \leq p_{i, j} \leq 1, \forall i \in \mathcal{K}, \forall j \in S_{i}, \\
& \sum_{j=1}^{\left|S_{i}\right|} p_{i, j}=1, \forall i \in \mathcal{K} .
\end{array}
$$

In the power allocation problem, the user structure and decoding order are obtained by the user association problem. Therefore, the optimization of power allocation coefficients is performed at the given user structure.

\section{Coalitional Game based User Association ALGORITHM}

In the considered multi-cell NOMA system, by dividing users into different clusters, each user is associated with the feasible BS which can provide a higher sum rate. Therefore, the formulated user association problem can be considered as a user clustering game $(\mathcal{N}, U)$ in coalition formation with non-transferable utility (NTU), since the coalition utility of any cluster is a mapping function and cannot be expressed as a function over the real line [32]. In user clustering game $(\mathcal{N}, U)$, the coalition utility of any cluster $S_{k}$ can be defined as follows:

$$
U\left(S_{k}\right)=\left\{\mathbf{u}\left(S_{k}\right) \in \mathbb{R}^{\left|S_{k}\right|} \mid u_{i}\left(S_{k}\right)=R_{k, i}, \forall i \in S_{k}\right\},
$$

where $u_{i}\left(S_{k}\right)$ is the payoff of the $i$-th user in cluster $S_{k}$, and $R_{k, i}$ is given by equation (13). According to [32], user clustering game $(\mathcal{N}, U)$ is in characteristic formation, since the payoff of any user only depends on the users in the same cluster.

In order to solve the user clustering game $(\mathcal{N}, U)$, two game theory based user association algorithms are proposed in this section. Firstly, preference relation based algorithm (PRA) provides a low-complexity solution. Secondly, simulated annealing based algorithm (SAA) is proposed for obtaining the global optimal solution.

\section{A. Preference Relation Based Algorithm}

In PRA, the users swap between different clusters based on their preference. In this paper, it is assumed that the users connect to different BSs by comparing the change of coalition utilities. In order to express the swap operation of users, a notation $\prec$ is introduced for denoting the preference. For any user $n$ in cluster $S_{i}$, the preference relation $S_{i} \prec_{n} S_{j}$ denotes that user $n$ is willing to be part of cluster $S_{j}$, rather than $S_{i}$, where $i \neq j$. The sum utility of clusters $S_{i}$ and $S_{j}$ is considered as the criteria for the swap operation. The strict preference relation in this paper is defined as follows:

$$
S_{i} \prec_{n} S_{j} \Leftrightarrow U\left(S_{i}\right)+U\left(S_{j}\right)<U\left(S_{i} \backslash\{n\}\right)+U\left(S_{j} \cup\{n\}\right) .
$$


In other words, user $n$ decides to split from cluster $S_{i}$ and join in cluster $S_{j}$, the sum utility of clusters $S_{i}$ and $S_{j}$ is strictly increased with the swap operation. In the proposed user association algorithm, the users compare the preference with all other clusters, and decide the swap operations based on the preference relation function. By introducing the compare-andswap operation, PRA is presented in Algorithm 1.

\begin{tabular}{l}
\hline Algorithm 1 Preference Relation based Algorithm (PRA) \\
\hline Initial State
\end{tabular}

The game is started from an initial structure $\mathcal{S}_{\text {init }}$, in which all users are associated with the nearest BSs.

Repeat

The users begin their operations from user 1 to user $N$.

For any user $n \in \mathcal{N}$, where $n \in S_{i}, S_{i} \in \mathcal{S}$,

1: User $n$ visits all other clusters from $S_{1}$ to $S_{K}$ except its current cluster. For any cluster $S_{j} \in \mathcal{S}$, where $j \neq i$.

2: Calculate the sum utilities of clusters $S_{i}$ and $S_{j}$.

3: User $n$ moves from cluster $S_{i}$ to $S_{j}$, the user structure is changed from $\mathcal{S}$ to $\mathcal{S}_{\text {new }}$.

4: All users in clusters $S_{i}$ and $S_{j}$ are ordered based on the optimal decoding order.

5: Calculate the new sum utility of clusters $S_{i}$ and $S_{j}$.

6: Compare the preference of user $n$ based on the strictly preference relation function (19).

7: If the preference relation $S_{i} \prec_{n} S_{j}$ is satisfied, record current partition as $\mathcal{S}=\mathcal{S}_{\text {new }}$.

8: Else, user $n$ moves to cluster $S_{i}$ and the user structure back to $\mathcal{S}$.

\section{Final State}

The game is finished when all of the users have no incentive to swap to other clusters.

The proposed algorithm can be started from any structure. For reducing the number of iterations in PRA, a simple scheme is considered as the initial partition, in which the users are connected to the nearest BSs. During the game, the users perform the compare-and-swap operations based on the sequence. If the last user has finished its operations, the first user starts the game again. In any user's round, the user visits all other clusters from $S_{1}$ to $S_{K}$. More particularly, the user disconnects from the associated BS, and connects to another BS. Note that the user's channel condition and interference are updated with the swap operation. At each iteration, the decoding order of the users in the changed clusters are re-ordered for applying SIC techniques. The sum utility of the changed clusters is calculated based on the closed-form expressions for power allocation coefficients, which will be discussed in Section V. If any user's swap operation is successfully executed, the user structure $\mathcal{S}$ is transformed to a new structure $\mathcal{S}_{\text {new }}$. The strategy $\sigma$ of any user $n$ in cluster $S_{i}$ is a set of possible operations:

$$
\sigma_{n}= \begin{cases}\mathcal{S} & \text { if } S_{i} \succ_{n} S_{j}, \forall S_{j} \in \mathcal{S}, \\ \mathcal{S}_{\text {new }} & \text { if } S_{i} \prec_{n} S_{j}, \exists S_{j} \in \mathcal{S} .\end{cases}
$$

If any user has visited all other clusters, its operations are finished, and the next user starts the compare-and-swap operations. The game is stopped until no user can join in other clusters, and therefore, the outputted user structure is the final structure $\mathcal{S}_{\text {final }}$.

\section{B. Simulated Annealing Based Algorithm}

In this subsection, $\mathrm{SAA}$ is proposed as a probabilistic approach for approximating the global optimum in a large number of possible user structures [33]. By randomly connecting users and BSs at each iteration, the global optimal solution can be obtained by the proposed algorithm. In SAA, the sum utility of all users in any structure is calculated for comparison. The sum utility of user structure $\mathcal{S}$ is calculated as follows:

$$
U(\mathcal{S})=\sum_{i=1}^{K} U\left(S_{i}\right)
$$

The initial user structure $\mathcal{S}_{\text {init }}$ is established by associating users with the nearest BSs. At this stage, a maximum utility $U_{\max }$ is defined as the sum utility of the initial structure. Durning the game, the new user structure $\mathcal{S}_{\text {new }}$ is produced by randomly connecting one user to one BS. The sum utility of the new structure $U\left(\mathcal{S}_{\text {new }}\right)$ is calculated. The maximum utility $U_{\max }$ and the user structure $\mathcal{S}$ are updated at each iteration. For the maximum utility $U_{\max }$, the value is compared with the sum utility of the new structure $U\left(\mathcal{S}_{\text {new }}\right)$, and replaced as follows:

$$
U\left(\mathcal{S}_{\text {new }}\right)>U_{\text {max }} \Rightarrow U_{\text {max }}=U\left(\mathcal{S}_{\text {new }}\right)
$$

In terms of the user structure, the acceptance of MetropolisHastings algorithm in [34] is adopted. If $U\left(\mathcal{S}_{\text {new }}\right)>U_{\max }$, the user structure is replaced by the new structure, i.e., $\mathcal{S}=$ $\mathcal{S}_{\text {new }}$. If $U\left(\mathcal{S}_{\text {new }}\right) \leq U_{\text {max }}$, the increment of the sum utility is obtained as follows:

$$
\Delta U\left(\mathcal{S}_{\text {new }}\right)=U\left(\mathcal{S}_{\text {new }}\right)-U_{\max },
$$

and the new structure is accepted at the following probability:

$$
\operatorname{Pr}=T e^{\left(\frac{\Delta U(\mathcal{S})}{U_{\max }}\right)},
$$

where $T$ is the temperature in simulated annealing methods. With the acceptance, maximum utility $U_{\max }$ is guaranteed to strictly increase during the proposed algorithm, while the user structure is capable of transforming to a worse neighbor structure. The probability for the current structure changes to a worse neighbor structure is based on the decrement of sum utility. If the gap between the current structure and the new structure is small, the probability is relatively large. If the sum utility is significantly reduced, the transformation almost never occurs. The acceptance ensures that the user structure always has a chance to jump out form a local optimum and converge to a global optimal partition with a large number of iterations. The proposed global optimal algorithm is presented in Algorithm 2.

In SAA, a maximum number of iterations $\Omega_{\max }$ is defined at the beginning of the algorithm. The sum utility in the algorithm is calculated by the optimal decoding order and the closed-form expressions for power allocation coefficients. The game is finished when the number of iterations $\Omega$ is equal to the maximum number of iterations $\Omega_{\max }$. As the 


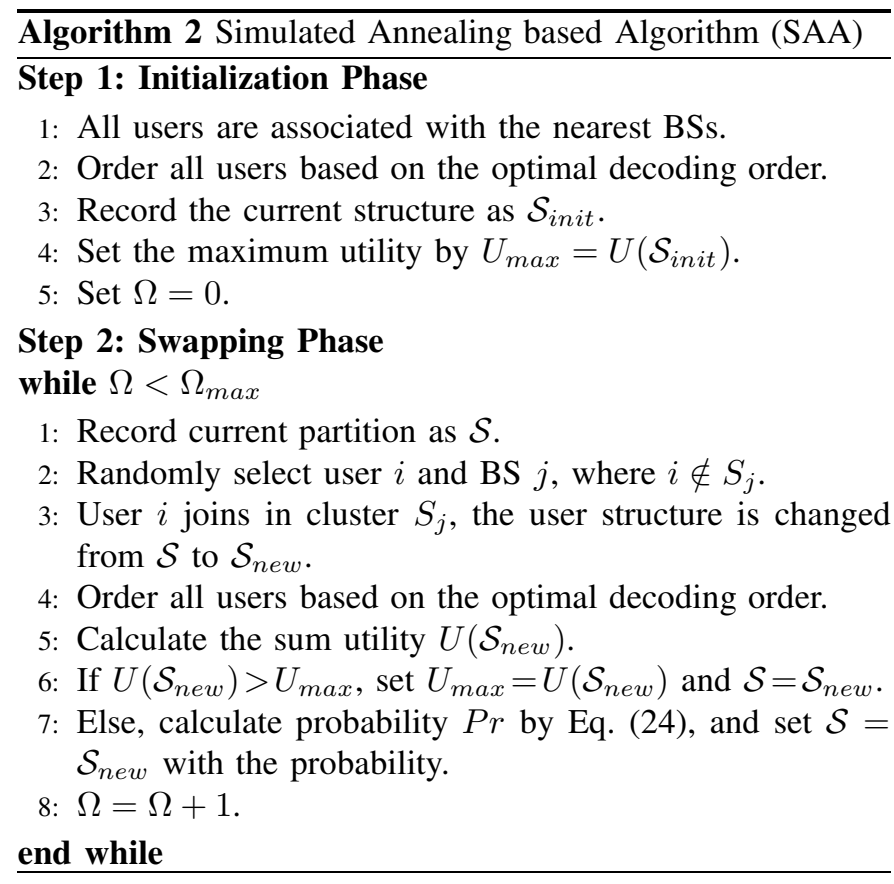

outcome of SAA, the enhanced sum rate can be obtained at the final iteration, e.g., the maximum utility $U_{\max }$. During the proposed algorithm, the maximum utility is guaranteed to be strictly increased. However, the final structure $\mathcal{S}$ at the $\Omega_{\max }$-th iteration may not be the enhanced structure, cause it may transform to a worse partition with the probability $\mathrm{Pr}$. Therefore, the final structure of SAA may not be a stable structure.

\section{Property Analysis of Proposed Algorithms}

In order to analyze the properties of the proposed algorithms, the complexity, convergence and stability of PRA are studied, and the optimality of SAA is discussed.

1) Complexity: In PRA, the users visit all other clusters for compare operations at each iteration, and execute swap operations if the preference relation conditions in (19) are satisfied. The game is repeated several times until it is finished, then the complexity of PRA is related to the number of cycles. One cycle means that the users have performed the compareand-swap operations for all clusters from user 1 to user $N$. By introducing the definition of cycles, the computational complexity of PRA can be given as follows:

Proposition 1. In PRA, at most $N(K-1)$ times of calculations are performed in each cycle. With a given number of cycle times $C$, the computational complexity of PRA is approximating $O(C N K)$.

Proof: In PRA, the complexity is based on the number of compare operations. Even though any user is not successfully moved to another cluster, the calculation is carried out for comparing the preference. In each cycle, one user need to visit all clusters except its current one, hence, $K-1$ clusters are visited by one user. For $N$ users, the compare operations in one cycle are performed $N(K-1)$ times. The total number of cycles is defined as $C$. Therefore, the computational complexity of PRA can be expressed as $O(C N K)$.
In terms of the global optimal algorithm, the computational complexity of SAA can be denoted by $O\left(\Omega_{\max }\right)$, since there is one time of calculation at each iteration and the total number of iterations is defined as $\Omega_{\max }$.

2) Convergence: The convergence of PRA for solving user clustering game $(\mathcal{N}, U)$ is guaranteed as follows:

Proposition 2. Starting at any initial user structure $\mathcal{S}_{\text {init }}$, user clustering game $(\mathcal{N}, U)$ with PRA is capable of converging to a final structure $\mathcal{S}_{\text {final }}$ which consists of a series of disjoint clusters.

Proof: In PRA, the users perform compare-and-swap operations for other clusters in order to maximize the sum rate. The user structure is changed only if one user decides to swap to another cluster, since the user is not moved with the compare operations. During swap operations, the user structure is transformed as follows:

$$
\mathcal{S}_{\text {init }} \rightarrow \mathcal{S}_{1} \rightarrow \mathcal{S}_{2} \cdots \rightarrow \mathcal{S}_{\text {final }}
$$

Assume that $\mathcal{S}_{a}$ is a user structure after $a$ times of swap operations in sequence (25), which includes $K$ clusters formed by $N$ users. From user structure $\mathcal{S}_{a}$ to the next partition $\mathcal{S}_{b}$, where $a<b$, one user is successfully moved from one cluster to another. More particular, the user is disconnect from one BS, and associated with a different one. The swap operation follows the preference relation condition in (19), which indicates that the sum utility of the changed clusters is strictly increased. Hence, the following condition is always satisfied for user structures $\mathcal{S}_{a}$ and $\mathcal{S}_{b}$ :

$$
\mathcal{S}_{a} \rightarrow \mathcal{S}_{b} \Leftrightarrow U\left(\mathcal{S}_{b}\right)>U\left(\mathcal{S}_{a}\right) .
$$

In other words, the sum utility is always increased with the transformation of user structures, as shown in sequence (25). With the finite number of users, the number of partitions is finite and equal to the Bell number [35]. Therefore, the sequence in (25) can always converge to a final structure $\mathcal{S}_{\text {final }}$. Note that any user can only join in one cluster at the same time, and hence, the final structure is formed by $K$ disjoint clusters.

The convergence of the simulated annealing based method is studied in [36]. It is proved that the algorithm based on simulated annealing with the acceptance of Metropolis procedure can converge to the optimal solution.

3) Stability: Given the convergence of PRA, the stability of the user association algorithm can be analyzed. In this paper, the stability of PRA follows Nash-stable in [37]. The definition is shown as follows:

Definition 1. A structure $\mathcal{S}$ is Nash-stable if $\forall n \in \mathcal{N}, n \in$ $S_{i}, S_{i} \in \mathcal{S}$, the preference relation $S_{i} \succ_{n} S_{j}$ is always satisfied for all $S_{j} \in \mathcal{S}, j \neq i$.

By introducing the definition of Nash-stable, the stability of PRA is guaranteed as follows:

Proposition 3. In user clustering game $(\mathcal{N}, U)$, any final user structure $\mathcal{S}_{\text {final }}$ resulting from PRA is a Nash-stable structure.

Proof: If the final partition $\mathcal{S}_{\text {final }}$ produced by PRA is not a Nash-stable structure, there exists at least one user $n$ 
tends to move to another cluster. Assume that user $n$ in cluster $S_{i}$ is willing be part of cluster $S_{j}$, then preference relation $S_{i} \prec_{n} S_{j}$ holds, where $i \neq j$. Based on the preference relation condition in (19), the sum utility of the clusters $S_{i}$ and $S_{j}$ is strictly increased by the swap operation of user $n$. There exists one partition $\mathcal{S}_{\text {new }}$ which satisfies $U\left(\mathcal{S}_{\text {new }}\right)>U\left(\mathcal{S}_{\text {final }}\right)$. It is contradicted to the fact that $\mathcal{S}_{\text {final }}$ is the final structure, since the sum utility of the final structure cannot be improved by swap operations. Therefore, the final structure achieved by PRA is Nash-stable.

4) Optimality: Note that the outcome of PRA is not guaranteed to converge to a global optimum. Alternatively, SAA is proposed for obtaining the global optimal solution. The optimality of SAA is shown as follows:

Proposition 4. With sufficiently iterations $\Omega_{\max }$, SAA can achieve the global optimum of the sum rate, and the result is Nash-stable.

Proof: In SAA, the final structure may not be the optimal result, since the user structure can be transformed to a partition with a deteriorated sum rate. However, the maximum utility $U_{\max }$ is guaranteed to strictly increased with the swap operations. It is proved in [38] and [39] that the algorithm based on simulated annealing method is capable of converging to a global optimal solution with the sufficient number of iterations. In a global optimal structure, the utility is maximized and the maximum utility $U_{\max }$ can not be increased. The user structure with the global optimal utility is also a Nash-stable structure since there is no user has the incentive to move to other clusters.

Note that the performance of SAA depends on the temperature $T$ and the maximum iterations $\Omega_{\max }$. More particular, the probability to accept a worse result is significantly affected by the temperature, and the result is improved with the increasing number of iterations. In particular, the sufficiently iterations of SAA is approximately exponential time. In this paper, the maximum iterations is set as a fixed number.

\section{Closed-Form Solutions for Power Allocation}

In this section, the power allocation problem (17) is solved by deriving closed-form solutions. Two situations, including the QoS constraint whether can be satisfied, are considered. Two closed-form expressions for the sum rate are respectively derived to achieve the global optimum in different situations.

Note that the proposed user clustering game $(\mathcal{N}, U)$ is in characteristic formation, the change of power allocation coefficients in any cluster will not affect the utility of other clusters since the inter-BS interference is not changed. Therefore, the power allocation optimization problem in (17) can be considered as $K$ problems for the individual clusters. For any cluster $S_{k}$ with $t$ users, the original problem can be equivalently transformed to the following problem:

$$
\begin{array}{cl}
\max _{\{\boldsymbol{p}\}} & \sum_{i=1}^{t} R_{k, i} \\
\text { s.t. } & R_{k, i} \geq \gamma, \forall i \in S_{k}, \\
& 0 \leq p_{k, i} \leq 1, \forall i \in S_{k}, \\
& \sum_{i=1}^{t} p_{k, i}=1 .
\end{array}
$$

In order to solve problem (27), the power allocation in [40] can be utilized. Note that the users in each cluster are ordered based on their channel conditions and inter-BS interferences, and the last user has the best channel quality. Therefore, the optimized power allocation in problem (27) is to achieve all users' target rates, and allocates the remaining power to the $t$-th user of cluster $S_{k}$ since it can utilize the power more efficiently $^{2}$. If constraint (27a) can be satisfied by all users in cluster $S_{k}$, a closed-form expression can be presented as follows:

Proposition 5. In power allocation optimization problem (27), the sum rate of users in any cluster $S_{k}$ is

$$
R\left(S_{k}\right)=t \gamma+\log _{2}\left\{1+\frac{1-\left(2^{\gamma}-1\right)\left[\sum_{i=1}^{t} \frac{\left(\rho I_{k, i}+1\right) 2^{(i-1) \gamma}}{\rho\left|h_{k, i}\right|^{2}}\right]}{\frac{\rho I_{k, t}+1}{\rho\left|h_{k, t}\right|^{2}}}\right\}
$$

if the following condition is satisfied:

$$
1-\left(2^{\gamma}-1\right)\left[\sum_{i=1}^{t} \frac{\left(\rho I_{k, i}+1\right) 2^{(i-1) \gamma}}{\rho\left|h_{k, i}\right|^{2}}\right] \geq 0 .
$$

Proof: See Appendix A.

It can be found that the derived closed-form expression (28) can be divided into two parts. The first part, $t \gamma$, is the QoS thresholds of all users in cluster $S_{k}$, and the second part is to allocate the remaining power to the $t$-th user. The inequality (29) is the condition for this case, which indicates all users in cluster $S_{k}$ can achieve the target rate. However, there exists another case that the transmit power cannot satisfy all users' QoS constraints in cluster $S_{k}$. In other words, with the given users and the decoding order, the constraint (27a) cannot hold for all users. In this case, users with the better channel conditions and less inter-BS interference have the higher priority to achieve the target rate, since less transmit power is consumed for satisfying these users' QoS constraints. Based on this strategy, the optimal power allocation in this case can be expressed as follows:

Proposition 6. In power allocation optimization problem (27), if the following condition holds:

$$
1-\left(2^{\gamma}-1\right)\left[\sum_{i=1}^{t} \frac{\left(\rho I_{k, i}+1\right) 2^{(i-1) \gamma}}{\rho\left|h_{k, i}\right|^{2}}\right]<0,
$$

\footnotetext{
${ }^{2}$ It is worth pointing out that reducing transmit power is capable of improving the outage probability of the considered multi-cell NOMA system, but this is beyond the scope of this paper.
} 
TABLE I: Table of Parameters

\begin{tabular}{|l|l|}
\hline The number of BSs & 4 \\
\hline Location of BS 1 & $\left(-0.4 R_{D} ; 0.4 R_{D}\right)$ \\
\hline Location of BS 2 & $\left(0.4 R_{D} ; 0.4 R_{D}\right)$ \\
\hline Location of BS 3 & $\left(-0.4 R_{D} ;-0.4 R_{D}\right)$ \\
\hline Location of BS 4 & $\left(0.4 R_{D} ;-0.4 R_{D}\right)$ \\
\hline System bandwidth & $10 \mathrm{MHz}$ \\
\hline Carrier frequency & $1 \mathrm{GHz}$ \\
\hline Thermal noise power & $\sigma^{2}=-174 \mathrm{dBm}$ \\
\hline Pass loss exponent & $\alpha=3$ \\
\hline The minimum data rate & $\gamma=0.1 \mathrm{Mbits} / \mathrm{s} / \mathrm{Hz}$ \\
\hline
\end{tabular}

there exists one user $n$ which satisfies the following conditions:

$$
\left\{\begin{array}{l}
\left(2^{\gamma}-1\right)\left[\sum_{i=n+1}^{t} \frac{\left(\rho I_{k, i}+1\right) 2^{(i-1) \gamma}}{\rho\left|h_{k, i}\right|^{2}}\right] \leq 1 \\
\left(2^{\gamma}-1\right)\left[\sum_{i=n}^{t} \frac{\left(\rho I_{k, i}+1\right) 2^{(i-1) \gamma}}{\rho\left|h_{k, i}\right|^{2}}\right]>1
\end{array}\right.
$$

The maximum sum rate of cluster $S_{k}$ is

$$
R\left(S_{k}\right)=(t-n) \gamma+\log _{2}\left[1+\frac{\left|h_{k, n}\right|^{2} \Delta p\left(S_{k}\right)}{\left|h_{k, n}\right|^{2}\left(1-\Delta p\left(S_{k}\right)\right)+I_{k, n}+\frac{1}{\rho}}\right],
$$

where $\Delta p\left(S_{k}\right)=1-\left(2^{\gamma}-1\right)\left[\sum_{i=n+1}^{t} \frac{\left(\rho I_{k, i}+1\right) 2^{(i-1) \gamma}}{\rho\left|h_{k, i}\right|^{2}}\right]$.

Proof: See Appendix B.

In above proposition, condition (30) indicates that the transmit power of BS $k$ cannot satisfy the QoS thresholds of all associated users. In this case, users after the $(n+1)$ th user (includes the $(n+1)$-th user) can achieve the target rate, and the remaining power cannot help at least one user to achieve the target rate, which corresponds to the conditions in inequality (31). As a result, the optimal power allocation of all users in cluster $S_{k}$ can be shown in (32).

By utilizing the closed-form expressions at each iteration of the proposed user association algorithms, the user association and power allocation problems are iteratively optimized.

\section{Simulation Results}

Simulation results are presented in this section to highlight the performance of the proposed user association algorithms and the derived closed-form expressions for power allocation. The Monte Carlo simulation is employed and the parameters are shown in Table I. Both OMA and fixed power NOMA schemes are provided as benchmarks. In terms of the OMA scheme, TDMA is adopted, where all users in TDMA schemes receive signals with the equally allocated time slots, and hence, the time slot allocation coefficient of any user is $1 / N$. For fixed power NOMA schemes, the power allocation coefficient of any user $n$ in cluster $S_{k}$ is considered as $p_{k, n}=\frac{\left|S_{k}\right|-n+1}{\mu}$, where $\mu=\sum_{i=1}^{\left|S_{k}\right|} i$ is utilized to ensure $\sum_{i=1}^{\left|S_{k}\right|} p_{k, i}=1$. By employing the the fixed power allocation coefficients, the individual rates and the outage probability of the users with weak channel conditions can be improved. The proposed user association algorithm, PRA, is also adopted in OMA and fixed power NOMA schemes, and the sum utilities are calculated based on the equal time slot allocation and the fixed power allocation, respectively.

In Fig. 2, the distance-based partition (DP) and PRA are compared, where the game is played 10 times in both schemes.

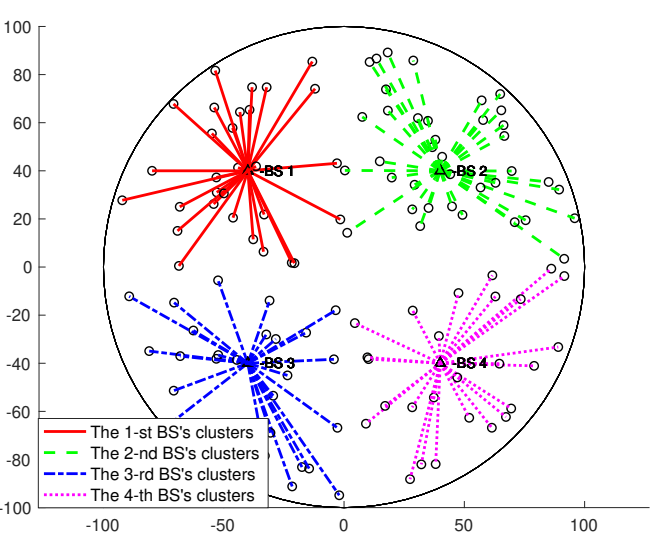

(a)

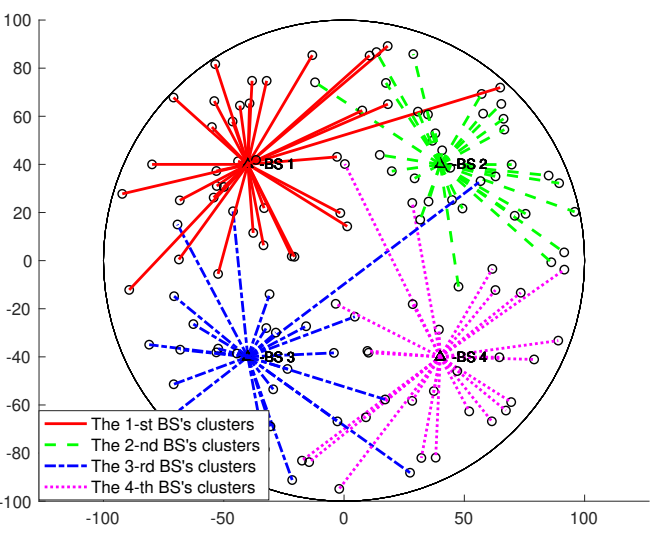

(b)

Fig. 2: An illustration of user association and power allocation. $N=12, R_{D}=100 \mathrm{~m}$, and $P_{t}=30 \mathrm{~dB}$. (a) The distance based partition with the optimized power allocation. (b) Stable partition based on PRA with the optimized power allocation.

Based on the optimal power allocation coefficients in propositions 5 and 6, from Fig. 2(a) to Fig. 2(b), the sum rate is increased from 17.4554 Mbits/s/Hz to $22.0703 \mathrm{Mbits} / \mathrm{s} / \mathrm{Hz}$, and the outage probability is reduced from 0.0333 to 0 . The outage probability is calculated by $P_{o}=E\left(N_{\text {fail }} / N\right)$, where $N_{\text {fail }}$ is the number of users cannot achieve the target rate $\gamma$. In Fig. 2(a), all users are associated with the nearest BSs. During PRA, several users are connected to the BS with large distances, as shown in Fig. 2(b). This is due to following reasons. Firstly, if any user cannot achieve its target rate when connecting to the nearest BS, it will be associated to the far BS which can provide enough power to satisfy the QoS threshold. It confirms to the fact that the outage probability is decreased. Secondly, if any cluster includes the user has an extremely good channel condition, the users with worse channel conditions will be allocated to other clusters, since more power can be allocated to the best user. As a result, such a strategy can increase the sum rate.

In Fig. 3, the performance of different schemes is simulated with a large range of transmit power, where DP and random partition (RP) are considered as benchmarks. It can 


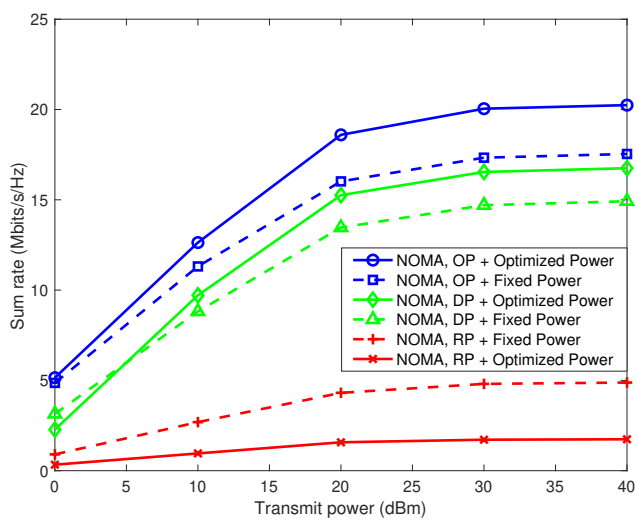

(a)

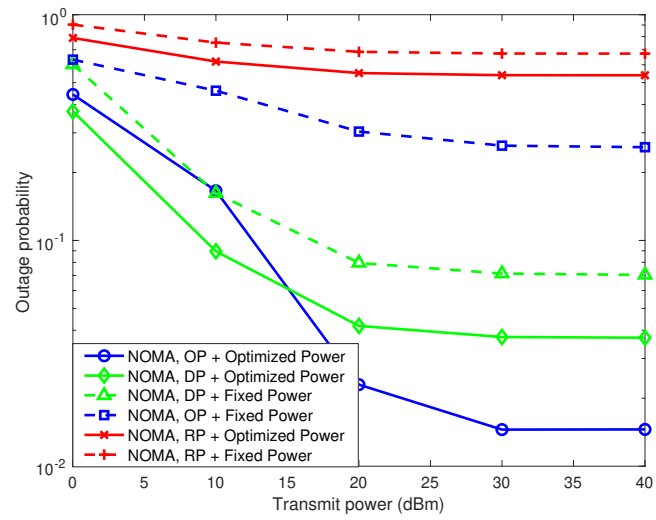

(b)

Fig. 3: The impact of transmit power for different schemes. $N=12$, and $R_{D}=100 \mathrm{~m}$. (a) Sum rate. (b) Outage probability.

be observed from Fig. 3(a) and Fig. 3(b) that PRA and power allocation are capable of significantly enhancing the sum rate and the outage probability for NOMA schemes, especially at high transmit power. By associating the users with the nearest BSs, both sum rate and outage probability can be greatly improved. The performance of the derived closedform expressions for power allocation can also be observed from the figure. It is worth pointing out that the outage probability of optimized partition (OP) is worse than DP with optimized power when the transmit power is between $0 \mathrm{dBm}$ and $15 \mathrm{dBm}$, since there is a trade-off between the sum rate and outage probability. In the overloaded system, some users cannot achieve their target rates at lower transmit power, then the performance of the proposed user association algorithm is saturated. At higher transmit power, the QoS constraints of these users can be satisfied, and hence, this saturation effect is avoided.

The effect of different radiuses is showed in Fig. 4, where both OMA and NOMA schemes are simulated. For any user, with the increasing radius of the disc, the average channel gain and interference channels are reduced. As a result, the performance including the sum rate and outage probability is deteriorated. It shows that the proposed user association algorithm and the derived closed-form solutions for power

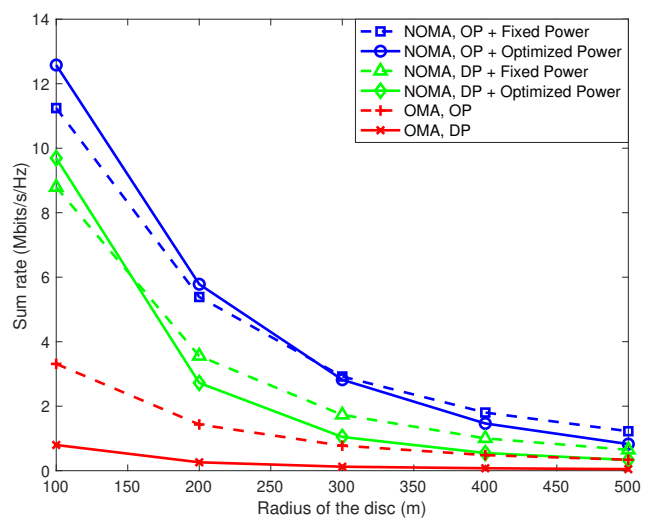

(a)

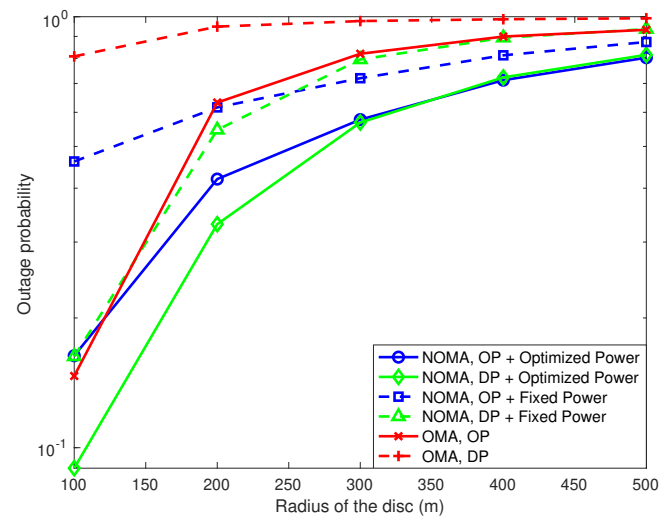

(b)

Fig. 4: The impact of different radiuses for NOMA and OMA schemes. $N=12$, and $P_{t}=10 \mathrm{~dB}$. (a) The distance based partition. (b) Stable partition based on PRA.

allocation can significantly improve the sum rate, and provides an acceptable outage probability. In Fig. 4(a), the NOMA scheme with optimized power is worse than fixed power when the radius is increased, since more power is allocated to the users with poor channel conditions for achieving the minimum data rate. For DP, this situation appears early, since the users in worse structures are more difficult to satisfy the QoS constraints. Another point is that the outage probability of OMA schemes with DP is raised faster than NOMA schemes. This is due to the fact that there is no interference in OMA schemes, and hence the influence of the channel gains becomes more obvious.

In Fig. 5, the sum rate and outage probability with different numbers of users are simulated. Both $3 \mathrm{BSs}$ and 4 BSs scenarios are considered, where the BSs are located at $\left(0 ; 0.5 R_{D}\right),\left(0.4 R_{D} ;-0.3 R_{D}\right)$, and $\left(-0.4 R_{D} ;-0.3 R_{D}\right)$ for the $3 \mathrm{BSs}$ scenario. It indicates that the sum rate and outage probability of NOMA schemes are improved when more BSs are included, while the OMA schemes are nearly not affected by the numbers of BSs. In NOMA schemes, the user with the strongest channel condition plays a dominant role, and hence, the sum rate will be increased since more BSs brings more of such users. However, in OMA schemes, there is no interference and the time slots are equally allocated, then 

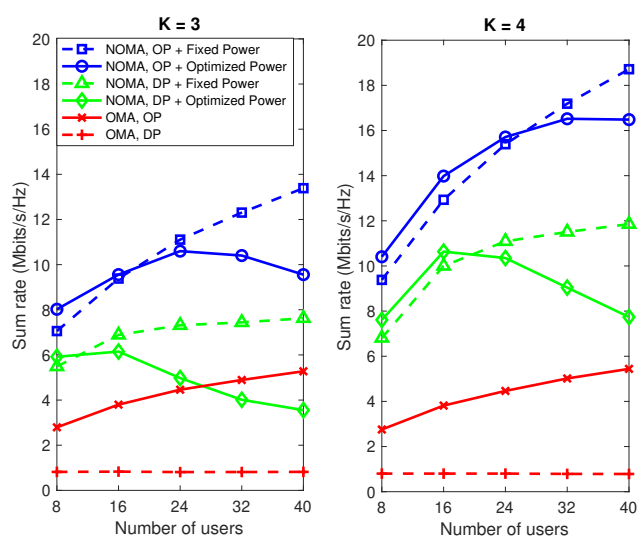

(a)
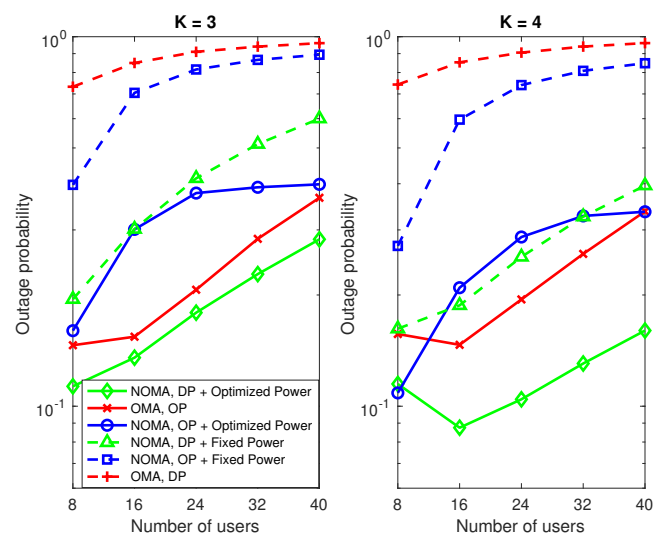

(b)

Fig. 5: The performance of NOMA and OMA schemes at different numbers of users. $R_{D}=100 \mathrm{~m}$, and $P_{t}=10 \mathrm{~dB}$. (a) Sum rate. (b) Outage probability.

the performance of both schemes are only affected by the number of users. It can also be observed from Fig. 5(a) that the sum rate of NOMA schemes with optimized power is less than that with fixed power when the users exceed a certain number. Note that the QoS constraint is not included in the user association problem, OP with the fixed power allocation may cause a OMA solution, in which the user with the best channel condition stay in the singleton cluster and the remaining users are allocated to a grand cluster. The sum rate can be improved in this case, while the outage probability is significantly increased. In optimal power allocation, the QoS threshold $\gamma$ is considered in the derived closed-from solutions, and thus this case can be avoid. However, the most power is allocated to the users with weaker channel conditions for achieving the target rate, which leads to a lower sum rate. Additionally, this certain number is decreased if a worse user structure is employed, which can be found by comparing OP with DP.

In Fig. 6, the iterations of the proposed algorithms are shown, in which fixed power allocation coefficients are adopted. In the imperfect decoding order scheme, the users in each cluster are not ordered based on the channel conditions and interference. The individual rate in this case is calculated by Eq. (8). Note that the performance and the complexity of

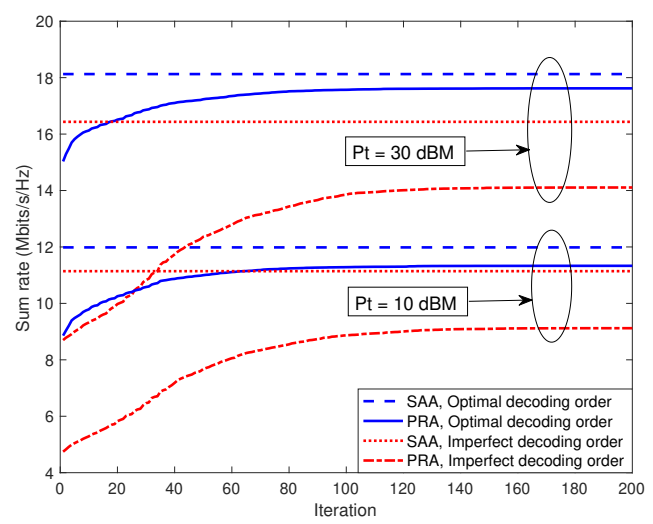

Fig. 6: The iterations of the user association algorithms with different decoding orders. $N=12$, and $R_{D}=100 \mathrm{~m}$.

SAA is significantly affected by the temperature and iteration numbers. According to [14], the temperature is set as $T=0.5$, and the maximum iterations is $\Omega_{\max }=10^{6}$. The flat lines are provided by SAA, which is proved to converge to the global optimum with fixed power allocation coefficients. The iterations of SAA is not shown, since it is $10^{6}$. Firstly, it can be observed that PRA is capable of achieving around $90 \%$ performance of the global optimum. Hence, the low complexity algorithm PRA can be regarded as a near-optimal algorithm. It is worth pointing out that there are 36 times of iterations in each cycle of PRA, which is confirmed to the analysis in Proposition 1 that $N(K-1)$ times of calculations are performed in each cycle. The sum utility of PRA can converge to a stable structure with approximately 100 times of iterations, which confirms the convergence and stability properties demonstrated in Proposition 2 and Proposition 3. Secondly, it is also indicated that the derived optimal decoding order can significantly enhance the convergence and final results of the proposed user association algorithms. Both PRA and SAA can achieve higher sum rates with the optimal decoding order than with the imperfect decoding order, and PRA with the imperfect decoding order needs around 150 times of iterations to reach the stable structure.

\section{CONCLUSIONS}

In this paper, a downlink multi-cell NOMA system with multiple BSs was investigated. In order to improve the sum rate of the proposed system, an optimization problem was formulated. The problem was divided into user association and power allocation problems. By considering the users associated with the same BS as one cluster, two user association algorithms were proposed to allocate the users to different clusters. PRA provided a low complexity near-optimal solution, while SAA obtained a global optimal outcome for the user association problem. For jointly solving the formulated problems, the closed-form expressions for power allocation were derived and performed at each iteration of the user association algorithms. The complexity, convergence, stability and optimality of the user association algorithms were analyzed. Simulation results showed that the proposed algorithms greatly improved the performance of the system in terms of the 
sum rate and the outage probability, and the user association scenario with NOMA schemes have a better performance than conversional OMA schemes. Moreover, incorporating the time slot or subcarrier allocation into the optimization problems is an important research direction for further enhancing the system performance.

\section{Appendix A: Proof of Proposition 5}

Proof: According to the QoS constraint, the data rate of the $n$-th user in cluster $S_{k}$ satisfies the following inequality:

$$
\log _{2}\left(1+\frac{\left|h_{k, n}\right|^{2} p_{k, n}}{\left|h_{k, n}\right|^{2} \sum_{i=n+1}^{t} p_{k, i}+I_{k, n}+\frac{1}{\rho}}\right) \geq \gamma,
$$

By introducing the minimal power allocation coefficient $\hat{p}$, the QoS constraint can be transformed as follows:

$$
\log _{2}\left(1+\frac{\left|h_{k, n}\right|^{2} \hat{p}_{k, n}}{\left|h_{k, n}\right|^{2} \sum_{i=n+1}^{t} \hat{p}_{k, i}+I_{k, n}+\frac{1}{\rho}}\right)=\gamma .
$$

The minimal power allocation coefficient of the $n$-th user in cluster $S_{k}$ can be expressed as

$$
\hat{p}_{k, n}=\left(2^{\gamma}-1\right)\left(\sum_{i=n+1}^{t} \hat{p}_{k, i}+\mathcal{A}_{k, n}\right),
$$

where $\mathcal{A}_{k, n}$ is the channel condition of the $n$-th user in cluster $S_{k}$, and defined as $\mathcal{A}_{k, n} \triangleq \frac{\rho I_{k, n}+1}{\rho\left|h_{k, n}\right|^{2}}$. The sum of all users' minimal power allocation coefficients in cluster $S_{k}$ can be obtained as follows:

$$
\begin{aligned}
\hat{p}\left(S_{k}\right) & =\sum_{i=1}^{t} \hat{p}_{k, i}=\left(2^{\gamma}-1\right) \sum_{i=1}^{t}\left(\sum_{j=i+1}^{t} \hat{p}_{k, j}+\frac{\rho I_{k, i}+1}{\rho\left|h_{k, i}\right|^{2}}\right) \\
& =\left(2^{\gamma}-1\right)\left[\sum_{i=1}^{t} 2^{(i-1) \gamma} \mathcal{A}_{k, i}\right] .
\end{aligned}
$$

By allocating the minimal power allocation coefficients to all users in cluster $S_{k}$, the remaining power allocation coefficient is given by

$$
\Delta p\left(S_{k}\right)=1-\hat{p}\left(S_{k}\right)=1-\left(2^{\gamma}-1\right)\left[\sum_{i=1}^{t} 2^{(i-1) \gamma} \mathcal{A}_{k, i}\right] .
$$

Eq. (35) can be equivalently transformed as follows:

$$
\begin{aligned}
& \hat{p}_{k, n}=\left(2^{\gamma}-1\right)\left(\sum_{i=n+1}^{t} \hat{p}_{k, i}+\mathcal{A}_{k, n}\right) \\
\Rightarrow & \left(2^{\gamma}-1\right)\left(\sum_{i=n+1}^{t} \Delta p_{k, i}\right)^{2}+\hat{p}_{k, n} \sum_{i=n+1}^{t} \Delta p_{k, i} \\
& =\left(2^{\gamma}-1\right)\left(\sum_{i=n+1}^{t} \Delta p_{k, i}\right)^{2}+\left(2^{\gamma}-1\right) \sum_{i=n+1}^{t} \Delta p_{k, i}\left(\sum_{i=n+1}^{t} \hat{p}_{k, i}+\mathcal{A}_{k, n}\right. \\
\Rightarrow & \sum_{i=n+1}^{t} \Delta p_{k, i}\left[\hat{p}_{k, n}+\left(2^{\gamma}-1\right) \sum_{i=n+1}^{t} \Delta p_{k, i}\right] \\
& =\left(2^{\gamma}-1\right) \sum_{i=n+1}^{t} \Delta p_{k, i}\left(\sum_{i=n+1}^{t} \Delta p_{k, i}+\sum_{i=n+1}^{t} \hat{p}_{k, i}+\mathcal{A}_{k, n}\right) \\
\Rightarrow & \frac{\hat{p}_{k, n}+\left(2^{\gamma}-1\right) \sum_{i=n+1}^{t} \Delta p_{k, i}}{\sum_{i=n+1}^{t} \Delta p_{k, i}+\sum_{i=n+1}^{t} \hat{p}_{k, i}+\mathcal{A}_{k, n}}=2^{\gamma}-1
\end{aligned}
$$

Based on Eq. (38), the QoS threshold can be expressed as

$$
\gamma=\log _{2}\left[1+\frac{\hat{p}_{k, n}+\left(2^{\gamma}-1\right) \sum_{i=n+1}^{t} \Delta p_{k, i}}{\sum_{i=n+1}^{t}\left(\hat{p}_{k, i}+\Delta p_{k, i}\right)+\mathcal{A}_{k, n}}\right]
$$

The increment of the individual rate for the $n$-th user is defined by $\Delta R_{k, n}=R_{k, n}-\gamma$. Based on Eq. (39), the increment of data rate at the $n$-th user can be expressed as follows:

$$
\begin{aligned}
\Delta R_{k, n} & =\log _{2}\left[1+\frac{\hat{p}_{k, n}+\Delta p_{k, n}}{\sum_{i=n+1}^{t}\left(\hat{p}_{k, i}+\Delta p_{k, i}\right)+\mathcal{A}_{k, n}}\right] \\
& -\log _{2}\left[1+\frac{\hat{p}_{k, n}+\left(2^{\gamma}-1\right) \sum_{i=n+1}^{t} \Delta p_{k, i}}{\sum_{i=n+1}^{t}\left(\hat{p}_{k, i}+\Delta p_{k, i}\right)+\mathcal{A}_{k, n}}\right] \\
& =\log _{2}\left(\frac{\sum_{i=n}^{t} \hat{p}_{k, i}+\sum_{i=n+1}^{t} \Delta p_{k, i}+\mathcal{A}_{k, n}+\Delta p_{k, n}}{\sum_{i=n}^{t} \hat{p}_{k, i}+2^{\gamma} \sum_{i=n+1}^{t} \Delta p_{k, i}+\mathcal{A}_{k, n}}\right) \\
& =\log _{2}\left[1+\frac{\Delta p_{k, n}-\left(2^{\gamma}-1\right) \sum_{i=n+1}^{t} \Delta p_{k, i}}{\sum_{i=n}^{t} \hat{p}_{k, i}+2^{\gamma} \sum_{i=n+1}^{t} \Delta p_{k, i}+\mathcal{A}_{k, n}}\right] .
\end{aligned}
$$

It can be found from Eq. (35) that the minimal power allocation coefficient for any user is fixed with the optimal decoding order. Therefore, the power allocation optimization problem in (27) can be equivalently transformed to a problem for maximizing the sum of increment data rates in cluster $S_{k}$, as shown in follows:

$$
\begin{aligned}
\max _{\{\boldsymbol{\Delta} \boldsymbol{p}\}} & \sum_{i=1}^{t} \Delta R_{k, i} \\
\text { s.t. } & 0 \leq \Delta p_{k, i} \leq 1, \forall i \in S_{k} \\
& \sum_{i=1}^{t} \Delta p_{k, i} \leq \Delta p\left(S_{k}\right)
\end{aligned}
$$

where $\Delta p$ is the collection of all increment power allocation coefficients. In problem (41), the QoS constraint in the original problem is removed, since all users have achieved the target rates in this case. It can be found from Eq. (40) that the optimal solution of problem (41) is that allocating all remaining power to the last user. If user $n$ in Eq. (40) is the last user, i.e., $n=t$, the value of $\Delta p_{k, n}$ is maximized, $\left(2^{\gamma}-1\right) \sum_{i=n+1}^{t} \Delta p_{k, i}$ is 0 , $\sum_{i=n}^{t} \hat{p}_{k, i}$ is minimized, and $2^{\gamma} \sum_{i=n+1}^{t} \Delta p_{k, i}$ is 0 . On the other hand, $\mathcal{A}_{k, n}$ is also minimized when $n=t$, since the value of $\mathcal{A}$ is ordered based on inequality (14). Therefore, the optimal power allocation in any cluster is that to satisfy all users' QoS constraints, then allocate all remaining power to the last user. With the optimal power allocation, the increment of the $t$-st user's individual rate is given by

$$
\begin{aligned}
\Delta R_{k, t} & =\log _{2}\left(1+\frac{\Delta p_{k, t}}{\mathcal{A}_{k, t}}\right)=\log _{2}\left(1+\frac{\Delta p\left(S_{k}\right)}{\mathcal{A}_{k, t}}\right) \\
& =\log _{2}\left\{1+\frac{1-\left(2^{\gamma}-1\right)\left[\sum_{i=1}^{t} 2^{(i-1) \gamma} \mathcal{A}_{k, i}\right]}{\mathcal{A}_{k, t}}\right\} .
\end{aligned}
$$


The optimal coalition utility of cluster $S_{k}$ can be expressed as

$$
R\left(S_{k}\right)=t \gamma+\log _{2}\left\{1+\frac{1-\left(2^{\gamma}-1\right)\left[\sum_{i=1}^{t} 2^{(i-1) \gamma} \mathcal{A}_{k, i}\right]}{\mathcal{A}_{k, t}}\right\},
$$

and the proposition is proved.

\section{ApPendix B: Proof of Proposition 6}

Proof: If condition (30) holds, the sum of all minimal power allocation coefficients $\hat{p}\left(S_{k}\right)$ is greater than 1 , hence, only part of users in cluster $S_{k}$ can achieve the target rates. Based on Eq. (35) and the optimal decoding order in (14), the following inequality can be obtained:

$$
\hat{p}_{k, 1}>\hat{p}_{k, 2}>\cdots>\hat{p}_{k, t} .
$$

The sequence of minimal power allocation coefficients indicates that the users with strong channel conditions are easier to achieve the target rates. Therefore, there exists one user $n$, where $1<n \leq t$, the QoS constraints of the users after the $(n+1)$-th user (includes the $(n+1)$-th user) can be satisfied, and the users before the $n$-th user (includes the $n$-th user) cannot achieve the target rates. The $n$-th user satisfies following conditions:

$$
\begin{aligned}
& \sum_{i=n+1}^{t} \hat{p}_{k, i} \leq 1, \\
& \sum_{i=n}^{t} \hat{p}_{k, i}>1 .
\end{aligned}
$$

Note that if $n=t$, it means that not user in cluster $S_{k}$ can achieve the target rate. By introducing Eq. (36), constraint (45) can equivalently transformed to

$$
\begin{aligned}
& \left(2^{\gamma}-1\right)\left[\sum_{i=n+1}^{t} 2^{(i-1) \gamma} \mathcal{A}_{k, i}\right] \leq 1, \\
& \left(2^{\gamma}-1\right)\left[\sum_{i=n}^{t} 2^{(i-1) \gamma} \mathcal{A}_{k, i}\right]>1 .
\end{aligned}
$$

By obtaining the $n$-th user in cluster $S_{k}$, the original power allocation optimization problem in (27) can be equivalently transformed to following problem:

$$
\begin{array}{cl}
\max _{\{\boldsymbol{p}\}} & \sum_{i=n}^{t} R_{k, i} \\
\text { s.t. } & R_{k, i} \geq \gamma, \forall i \in[n, t], \\
& 0 \leq p_{k, i} \leq 1, \forall i \in[n, t], \\
& \sum_{i=n}^{t} p_{k, i} \leq 1 .
\end{array}
$$

In order to obtain the optimal solution for problem (47), the power allocated to the users after the $(n+1)$-user (includes the $(n+1)$-th user) for satisfying the QoS thresholds, and all remaining power is allocated to the $n$-th user. The remaining power allocation coefficient is given by

$$
\Delta p\left(S_{k}\right)=1-\left(2^{\gamma}-1\right)\left[\sum_{i=n+1}^{t} 2^{(i-1) \gamma} \mathcal{A}_{k, i}\right] .
$$

By allocating all remaining power to the $n$-th user, the data rate for the $n$-th user can be expressed as follows:

$$
R_{k, n}=\log _{2}\left[1+\frac{\left|h_{k, n}\right|^{2} \Delta p\left(S_{k}\right)}{\left|h_{k, n}\right|^{2}\left(1-\Delta p\left(S_{k}\right)\right)+I_{k, n}+\frac{1}{\rho}}\right] .
$$

Therefore, the optimal sum rate of cluster $S_{k}$ is

$$
R\left(S_{k}\right)=(t-n) \gamma+\log _{2}\left[1+\frac{\left|h_{k, n}\right|^{2} \Delta p\left(S_{k}\right)}{\left|h_{k, n}\right|^{2}\left(1-\Delta p\left(S_{k}\right)\right)+I_{k, n}+\frac{1}{\rho}}\right],
$$

and the proof is completed.

\section{REFERENCES}

[1] K. Wang, Y. Liu, Z. Ding, and A. Nallanathan, "User association in nonorthogonal multiple access networks," in IEEE Proc. of International Commun. Conf. (ICC), May 2018, pp. 1-6.

[2] P. Wang, J. Xiao, and L. P, "Comparison of orthogonal and nonorthogonal approaches to future wireless cellular systems," IEEE Veh. Technol. Mag., vol. 1, no. 3, pp. 4-11, Sept. 2006.

[3] Y. Liu, Z. Qin, M. Elkashlan, Z. Ding, A. Nallanathan, and L. Hanzo, "Nonorthogonal multiple access for 5G and beyond," Proceedings of the IEEE, vol. 105 , no. 12 , pp. 2347-2381, Dec. 2017.

[4] Z. Ding, X. Lei, G. K. Karagiannidis, R. Schober, J. Yuan, and V. Bhargava, "A survey on non-orthogonal multiple access for $5 \mathrm{G}$ networks: Research challenges and future trends," IEEE J. Sel. Areas Commun., vol. PP, no. 99, pp. 1-1, 2017.

[5] Z. Ding, Y. Liu, J. Choi, Q. Sun, M. Elkashlan, C. L. I, and H. V. Poor, "Application of non-orthogonal multiple access in LTE and 5G networks," IEEE Commun. Mag., vol. 55, no. 2, pp. 185-191, Feb. 2017.

[6] Y. Cai, Z. Qin, F. Cui, G. Y. Li, and J. A. McCann, "Modulation and Multiple Access for 5G Networks," ArXiv e-prints, Feb. 2017.

[7] Y. Saito, A. Benjebbour, Y. Kishiyama, and T. Nakamura, "Systemlevel performance of downlink non-orthogonal multiple access (NOMA) under various environments," in 2015 IEEE 81st Vehicular Technology Conference (VTC Spring), May 2015, pp. 1-5.

[8] Z. Ding, Z. Yang, P. Fan, and H. V. Poor, "On the performance of non-orthogonal multiple access in 5G systems with randomly deployed users," IEEE Signal Process. Lett., vol. 21, no. 12, pp. 1501-1505, Dec. 2014.

[9] P. Xu, Z. Ding, X. Dai, and H. V. Poor, "A new evaluation criterion for non-orthogonal multiple access in 5G software defined networks," IEEE Access, vol. 3, pp. 1633-1639, 2015.

[10] Z. Ding, P. Fan, and V. Poor, "Impact of user pairing on 5G nonorthogonal multiple access downlink transmissions," IEEE Trans. Veh. Technol., vol. PP, no. 99, pp. 1-1, 2015.

[11] S. Timotheou and I. Krikidis, "Fairness for non-orthogonal multiple access in 5G systems," IEEE Signal Process. Lett., vol. 22, no. 10, pp. 1647-1651, Oct. 2015.

[12] W. Liang, Z. Ding, Y. Li, and L. Song, "User pairing for downlink nonorthogonal multiple access networks using matching algorithm," IEEE Trans. Commun., vol. 65, no. 12, pp. 5319-5332, Dec. 2017.

[13] Y. Liu, M. Elkashlan, Z. Ding, and G. K. Karagiannidis, "Fairness of user clustering in MIMO non-orthogonal multiple access systems," IEEE Commun. Lett., vol. 20, no. 7, pp. 1465-1468, July 2016.

[14] B. Di, L. Song, and Y. Li, "Sub-channel assignment, power allocation, and user scheduling for non-orthogonal multiple access networks," IEEE Trans. Wireless Commun., vol. 15, no. 11, pp. 7686-7698, Nov. 2016.

[15] M. S. Ali, H. Tabassum, and E. Hossain, "Dynamic user clustering and power allocation for uplink and downlink non-orthogonal multiple access (NOMA) systems," IEEE Access, vol. 4, pp. 6325-6343, 2016.

[16] L. P. Qian, Y. Wu, H. Zhou, and X. Shen, "Dynamic cell association for non-orthogonal multiple-access V2S networks," IEEE J. Sel. Areas Commun., vol. 35, no. 10, pp. 2342-2356, Oct. 2017.

[17] — "Joint uplink base station association and power control for small-cell networks with non-orthogonal multiple access," IEEE Trans. Wireless Commun., vol. 16, no. 9, pp. 5567-5582, Sept. 2017.

[18] W. Shin, M. Vaezi, B. Lee, D. J. Love, J. Lee, and H. V. Poor, "Nonorthogonal multiple access in multi-cell networks: Theory, performance, and practical challenges," IEEE Commun. Mag., vol. 55, no. 10, pp. 176-183, Oct. 2017.

[19] K. S. Ali, H. Elsawy, A. Chaaban, and M. S. Alouini, "Non-orthogonal multiple access for large-scale 5G networks: Interference aware design," IEEE Access, vol. 5, pp. 21 204-21 216, 2017.

[20] Y. Liu, Z. Qin, M. Elkashlan, A. Nallanathan, and J. A. McCann, "Nonorthogonal multiple access in large-scale heterogeneous networks," IEEE J. Sel. Areas Commun., vol. 35, no. 12, pp. 2667-2680, Dec. 2017.

[21] A. S. Marcano and H. L. Christiansen, "Impact of NOMA on network capacity dimensioning for $5 \mathrm{G}$ hetnets," IEEE Access, vol. 6, pp. 13587 13603,2018 
[22] D. Ni, L. Hao, Q. T. Tran, and X. Qian, "Power allocation for downlink noma heterogeneous networks," IEEE Access, vol. 6, pp. 26742-26 752, 2018.

[23] M. S. Ali, E. Hossain, and D. I. Kim, "Coordinated multipoint transmission in downlink multi-cell NOMA systems: Models and spectral efficiency performance," IEEE Wireless Commun., vol. 25, no. 2, pp. 24-31, Apr. 2018.

[24] M. S. Ali, E. Hossain, A. Al-Dweik, and D. I. Kim, "Downlink power allocation for CoMP-NOMA in multi-cell networks," IEEE Transactions on Communications, vol. 66, no. 9, pp. 3982-3998, Sep. 2018.

[25] Y. Sun, Z. Ding, and X. Dai, "On the performance of downlink NOMA in multi-cell mmwave networks," IEEE Commun. Lett., vol. 22, no. 11, pp. 2366-2369, Nov. 2018.

[26] Y. Fu, Y. Chen, and C. W. Sung, "Distributed power control for the downlink of multi-cell NOMA systems," IEEE Trans. Wireless Commun., vol. 16, no. 9, pp. 6207-6220, Sept. 2017.

[27] L. You, D. Yuan, L. Lei, S. Sun, S. Chatzinotas, and B. Ottersten, "Resource optimization with load coupling in multi-cell NOMA," IEEE Trans. Wireless Commun., vol. 17, no. 7, pp. 4735-4749, July 2018.

[28] J. Cui, Y. Liu, Z. Ding, P. Fan, and A. Nallanathan, "Qoe-based resource allocation for multi-cell NOMA networks," IEEE Trans. Wireless Commun., vol. 17, no. 9, pp. 6160-6176, Sept. 2018.

[29] Y. Saito, Y. Kishiyama, A. Benjebbour, T. Nakamura, A. Li, and K. Higuchi, "Non-orthogonal multiple access (NOMA) for cellular future radio access," in 2013 IEEE 77th Vehicular Technology Conference (VTC Spring), June 2013, pp. 1-5.

[30] R. M. Karp, "Reducibility among combinatorial problems," in Complexity of computer computations. Springer, 1972, pp. 85-103.

[31] M. R. Garey and D. S. Johnson, Computers and intractability. wh freeman New York, 2002, vol. 29.

[32] Z. Han, Game theory in wireless and communication networks: theory, models, and applications. Cambridge University Press, 2012.

[33] S. Kirkpatrick, C. D. Gelatt, M. P. Vecchi et al., "Optimization by simulated annealing," science, vol. 220, no. 4598, pp. 671-680, 1983.

[34] N. Metropolis, A. W. Rosenbluth, M. N. Rosenbluth, A. H. Teller, and E. Teller, "Equation of state calculations by fast computing machines," The journal of chemical physics, vol. 21, no. 6, pp. 1087-1092, 1953.

[35] D. Ray, A game-theoretic perspective on coalition formation. Oxford University Press, 2007.

[36] V. Granville, M. Krivanek, and J. P. Rasson, "Simulated annealing: a proof of convergence," IEEE Transactions on Pattern Analysis and Machine Intelligence, vol. 16, no. 6, pp. 652-656, June 1994.

[37] A. Bogomolnaia and M. O. Jackson, "The stability of hedonic coalition structures," Games and Economic Behavior, vol. 38, no. 2, pp. 201-230, 2002.

[38] L. Davis, Genetic algorithms and simulated annealing. Morgan Kaufman Publishers, Inc., Los Altos, CA, 1987.

[39] O. Häggström, Finite Markov chains and algorithmic applications. Cambridge University Press, 2002, vol. 52.

[40] Z. Chen, Z. Ding, X. Dai, and R. Zhang, "An optimization perspective of the superiority of NOMA compared to conventional OMA," IEEE Trans. Signal Process., vol. 65, no. 19, pp. 5191-5202, Oct. 2017.

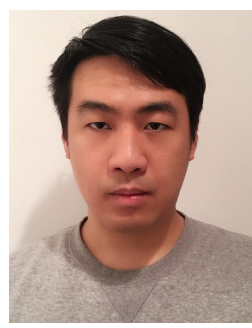

Kaidi Wang (S'16) received the MS degree in communications and signal processing from Newcastle University, UK, in 2014. He is currently working toward the $\mathrm{PhD}$ degree in wireless communication with the University of Manchester, UK. He was with Lancaster University from 2015 to 2018 . His current research interests include game theory, convex optimization, non-orthogonal multiple access, and 5G wireless networks.

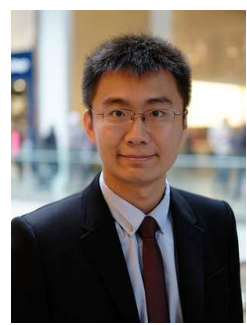

2017.

His research interests include $5 \mathrm{G}$ and beyond wireless networks, the Internet of Things, machine learning, and stochastic geometry. He has served as a TPC Member for many IEEE conferences, such as GLOBECOM and ICC. He received the Exemplary Reviewer Certificate of IEEE WIRELESS COMMUNICATIONS LETTERS in 2015, IEEE TRANSACTIONS ON COMMUNICATIONS in 2016 and 2017, and IEEE TRANSACTIONS ON WIRELESS COMMUNICATIONS in 2017 and 2018. He has served as the Publicity Co-Chair for VTC 2019-Fall. He is currently an Editor on the Editorial Board of the IEEE TRANSACTIONS ON COMMUNICATIONS, IEEE COMMUNICATIONS LETTERS, and IEEE ACCESS. He also serves as a Guest Editor for IEEE JSTSP special issue on Signal Processing Advances for Non-Orthogonal Multiple Access in Next Generation Wireless Networks.

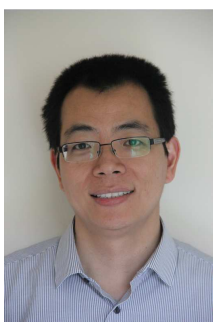

Princeton University.

Dr Ding' research interests are 5G networks, game theory, cooperative and energy harvesting networks and statistical signal processing. He is serving as an Editor for IEEE Transactions on Communications, IEEE Transactions on Vehicular Technology, and Journal of Wireless Communications and Mobile Computing, and was an Editor for IEEE Wireless Communication Letters, IEEE Communication Letters from 2013 to 2016. He received the best paper award in IET ICWMC-2009 and IEEE WCSP-2014, the EU Marie Curie Fellowship 2012-2014, the Top IEEE TVT Editor 2017, IEEE Heinrich Hertz Award 2018, IEEE Jack Neubauer Memorial Award 2018 and IEEE Best Signal Processing Letter Award 2018. 


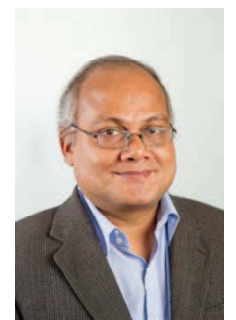

Arumugam Nallanathan (S'97-M'00-SM'05-F'17) is Professor of Wireless Communications and Head of the Communication Systems Research (CSR) group in the School of Electronic Engineering and Computer Science at Queen Mary University of London since September 2017. He was with the Department of Informatics at King's College London from December 2007 to August 2017, where he was Professor of Wireless Communications from April 2013 to August 2017 and a Visiting Professor from September 2017. He was an Assistant Professor in the Department of Electrical and Computer Engineering, National University of Singapore from August 2000 to December 2007. His research interests include 5G Wireless Networks, Internet of Things (IoT) and Molecular Communications. He published nearly 500 technical papers in scientific journals and international conferences. He is a co-recipient of the Best Paper Awards presented at the IEEE International Conference on Communications 2016 (ICC'2016) , IEEE Global Communications Conference 2017 (GLOBECOM'2017) and IEEE Vehicular Technology Conference 2018 (VTC'2018). $\mathrm{He}$ is an IEEE Distinguished Lecturer. He has been selected as a Web of Science Highly Cited Researcher in 2016.

$\mathrm{He}$ is an Editor for IEEE Transactions on Communications. He was an Editor for IEEE Transactions on Wireless Communications (2006-2011), IEEE Transactions on Vehicular Technology (2006-2017), IEEE Wireless Communications Letters and IEEE Signal Processing Letters. He served as the Chair for the Signal Processing and Communication Electronics Technical Committee of IEEE Communications Society and Technical Program Chair and member of Technical Program Committees in numerous IEEE conferences. He received the IEEE Communications Society SPCE outstanding service award 2012 and IEEE Communications Society RCC outstanding service award 2014.

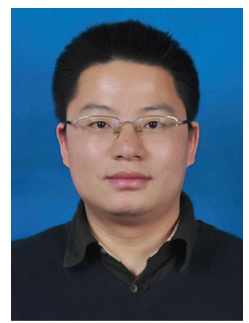

Mugen Peng (M'05-SM'11) received the Ph.D degree in communication and information systems from the Beijing University of Posts and Telecommunications (BUPT), Beijing, China, in 2005. Afterward, he joined BUPT, where he has been a Full Professor with the School of Information and Communication Engineering since 2012. He has authored and co-authored over 100 refereed IEEE journal papers and over 300 conference proceeding papers. His main research areas include wireless communication theory, radio signal processing, cooperative communication, self-organization networking, heterogeneous networking, cloud communication, and Internet of Things. Dr. Peng was a recipient of the 2018 Heinrich Hertz Prize Paper Award, the 2014 IEEE ComSoc AP Outstanding Young Researcher Award, and the Best Paper Award in the JCN 2016, IEEE WCNC 2015, IEEE GameNets 2014, IEEE CIT 2014, ICCTA 2011, ICBNMT 2010, and IET CCWMC 2009. He is currently or have been on the Editorial/Associate Editorial Board of the IEEE Communications Magazine, IEEE ACCESS, IEEE Internet of Things Journal, IET Communications, and China Communications. 